\title{
NUMERICAL SIMULATION OF A POINT-SOURCE INITIATED FLAME BALL WITH HEAT LOSSES
}

\author{
Jacques Audounet ${ }^{1}$, Jean-Michel Roquejoffre ${ }^{1}$ and HÉlène Rouzaud ${ }^{1}$
}

\begin{abstract}
This article is devoted to the numerical study of a flame ball model, derived by Joulin, which obeys to a singular integro-differential equation. The numerical scheme that we analyze here, is based upon a one step method, and we are interested in its long-time behaviour. We recover the same dynamics as in the continuous case: quenching, or stabilization of the flame, depending on heat losses, and an energy input parameter.
\end{abstract}

Mathematics Subject Classification. 35A40, 45J05, 65M12.

Received: July 13, 2001. Revised: January 14, 2002.

\section{INTRODUCTION}

The purpose of this work is the study of a numerical scheme allowing the observation of the large time dynamics of a flame ball model. This model is derived in [5] through high activation energy asymptotics: the time-evolution of the radius $R(t)$ of this spherical flame is governed by the fractional differential equations:

$$
R \partial_{1 / 2} R=R \log R+E q-\lambda R^{3}, \quad R(0)=0,
$$

where $\partial_{1 / 2}$ denotes the half-time derivative:

$$
\partial_{1 / 2} R:=\frac{1}{\sqrt{\pi}} \int_{0}^{t} \frac{\dot{R}(s)}{\sqrt{t-s}} \mathrm{~d} s=\frac{1}{\sqrt{\pi}} \frac{\mathrm{d}}{\mathrm{d} t} \int_{0}^{t} \frac{R(s)}{\sqrt{t-s}}
$$

The function $q$ is a smooth non-negative function defined on $\mathbb{R}_{+}$, with unit total mass; it measures the timevariation of the energy input; the positive parameters $E$ and $\lambda$ represent respectively the intensities of the heat source and of radiative heat losses.

In [12], it is proved that there exists a critical value $\lambda_{\text {cr }}$ such that the flame always quenches if $\lambda>\lambda_{\text {cr }}$; if $\lambda<\lambda_{\text {cr }}$, we have, as in [1], the existence of a critical energy $E_{\mathrm{cr}}(q, \lambda)$ such that the flame quenches if $E<E_{\mathrm{cr}}$; it stabilizes to $R_{2}$ (resp. $R_{1}$ ) if $E>E_{\text {cr }}$ (resp. $E=E_{\text {cr }}$ ), where $R_{2}>R_{1}$ are the solutions of the equation:

$$
\log R=\lambda R^{2} .
$$

Keywords and phrases. Flame ball, integro-differential equation, time discretisation, numerical quenching.

1 UFR MIG, UMR CNRS 5640, Université Paul Sabatier, 118 route de Narbonne, 31062 Toulouse Cedex, France.

e-mail: roque@mip.ups-tlse.fr 
This phenomenon has already been represented by Joulin [8] in the case $\lambda=0$. The scheme he used was based upon the integro-differential equation (0.1): a discretisation of $\dot{R}$ was performed in a space of piecewise constant or linear functions. Here instabilities appear in the long times, and even in the short times if we consider the approximation of $\dot{R}$ by a piecewise linear function.

The numerical scheme that we present here is based on a time-discretisation of the diffusive formulation, used in [1] and [12] for the mathematical study of this problem: the radius $R(t)$ can be expressed as the trace at $x=0$ of the semi-linear parabolic equation:

$$
\left\{\begin{array}{l}
u_{t}-u_{x x}=2 \delta_{x=0}\left(\log u+\frac{E q}{u}-\lambda u^{2}\right), x \in \mathbb{R} \\
u(0, .)=0 .
\end{array}\right.
$$

This formulation has been used in [7] to study Volterra integral equations, and here it is essential to characterize the long-time behaviour of the flame. In particular, a fundamental tool for this study is the existence of a maximum principle for (0.3). The time-discretisation we apply here has the advantage to preserve this maximum principle and we use it to prove that our scheme conserves the dynamics of solution, namely the threshold phenomena on the parameters $E$ and $\lambda$.

This paper is divided in four sections. In the first one, we present the scheme under consideration; in particular, we define the notion of numerical quenching of the flame. In Section 2, we prove the convergence of this scheme for $O(1)$ times. This part is rather standard, except the fact we push the analysis to the vicinity of quenching times. Nevertheless, we will need the convergence result for the dynamical study of the next section in which we prove by a shooting method that we recover with the numerical scheme all the qualitative behaviours expected. In the last section, we present numerical simulations and we compare this method with the time-discretisation used in [8].

\section{Presentation of the scheme}

We consider the equation:

$$
R \partial_{1 / 2} R=R \log R+E q-\lambda R^{3}, t \in \mathbb{R}_{+}, R(0)=0
$$

The radius $R$ can be seen $(c f .[1])$ as the trace on the axis $x=0$ of the solution $u$ of the diffusive problem:

$$
\left\{\begin{array}{l}
u_{t}-u_{x x}=2 \delta_{x=0}\left(\log u+\frac{E q}{u}-\lambda u^{2}\right), x \in \mathbb{R} \\
u(0, .)=0
\end{array}\right.
$$

which is in turn equivalent to:

$$
\left\{\begin{array}{l}
u_{t}-u_{x x}=0, x>0 \\
u_{x}(t, 0)=-\log u(t, 0)-\frac{E q(t)}{u(t, 0)}+\lambda u(t, 0)^{2} \\
u(0, .)=0 .
\end{array}\right.
$$

Numerical methods for this type of integro-differential equations have been presented by Brunner [4]. For example, diffusive problems can be discretized by multisteps methods (see [10] and [11]). But that fails here, because of the diffusive character of the problem, and of its non linear, non lipschitz singularity. That is why it is approximated in [2] via a time discretisation based upon the implicit Euler scheme. Applying here this 
scheme, with $\tau$ denoting the time step of the discretisation, we get:

$$
\left\{\begin{array}{l}
\frac{u^{n+1}-u^{n}}{\tau}-u_{x x}^{n+1}=0, x>0 \\
u_{x}^{n+1}(0)=-\log u^{n+1}(0)-\frac{E q^{n+1}}{u^{n+1}(0)}+\lambda u^{n+1}(0)^{2} \\
u^{0}=0
\end{array}\right.
$$

where $q^{n}=q(n \tau)$.

First, by induction, and because of the maximum principle, we have $u^{n} \geq 0$. Then, let us remark that (1.4) determines explicitly the quantity in which we are really interested, i.e. the sequence $R^{n}:=u^{n}(0)$.

Indeed, if $\hat{u}^{n}$ is the Fourier transform of $u^{n}$ :

$$
\hat{u}^{n}(t)=\int_{\mathbb{R}} u^{n}(x) \mathrm{e}^{-2 i \pi t x} \mathrm{~d} x,
$$

this semi-discrete scheme is equivalently formulated under the form:

$$
\left\{\begin{array}{l}
\hat{u}^{n+1}=\frac{1}{1+4 \pi^{2} \xi^{2} \tau} \hat{u}^{n}+\frac{2 \tau}{1+4 \pi^{2} \xi^{2} \tau} f^{n+1}\left(u^{n+1}(0)\right) \\
\hat{u}^{0}=0
\end{array}\right.
$$

which yields, with $f^{n+1}(r)=\log r+\frac{E q^{n+1}}{r}-\lambda r^{2}$ :

$$
\hat{u}^{n+1}(\xi)=\frac{\hat{u}^{0}(\xi)}{\left(1+4 \pi^{2} \xi^{2} \tau\right)^{n+1}}+2 \tau \sum_{k=0}^{n} \frac{1}{\left(1+4 \pi^{2} \xi^{2} \tau\right)^{k}} f^{n+1-k}\left(u^{n+1-k}(0)\right) .
$$

This defines $u^{n+1}(0)$ as the solution of:

$$
\left\{\begin{array}{l}
u^{n+1}(0)=\int_{\mathbb{R}} \frac{1}{1+4 \pi^{2} \xi^{2} \tau} \hat{u}^{n}(\xi) \mathrm{d} \xi+\sqrt{\tau} f^{n+1}\left(u^{n+1}(0)\right) \\
u^{0}=0
\end{array} .\right.
$$

Now searching $R^{n}=u^{n}(0)$ under the form $R^{n}=\alpha^{n}+\sqrt{\tau} f^{n}\left(u^{n}(0)\right)=\alpha^{n}+\sqrt{\tau} g^{n}$, with $\alpha^{n}+\sqrt{\tau} g^{n}>0$, we get:

$$
\left\{\begin{array}{l}
\alpha^{n}=\int_{\mathbb{R}} \frac{\hat{u}^{n-1}(\xi) \mathrm{d} \xi}{1+4 \pi^{2} \xi^{2} \tau}=\sqrt{\tau} \sum_{k=1}^{n-1} \theta_{n-k+1} g^{k}, \quad \hat{u}^{0}=0 \\
g^{n}=f^{n}\left(\alpha^{n}+\sqrt{\tau} g^{n}\right), \quad n \geq 1
\end{array}\right.
$$

where

$$
\theta_{p+1}=\int_{\mathbb{R}} \frac{2 \sqrt{\tau}}{\left(1+4 \pi^{2} \xi^{2} \tau\right)^{p+1}} \mathrm{~d} \xi=\frac{2 p-1}{2 p} \theta_{p}=\frac{C_{2 p-1}^{p}}{2^{2 p-1}} \theta_{1}, \text { with } \theta_{1}=1 .
$$

Thus, the determination of the sequence $\left(R^{n}\right)$ consists in successive resolutions of:

$$
\phi_{n}\left(g^{n}\right):=g^{n}-\log \left(\alpha^{n}+\sqrt{\tau} g^{n}\right)-\frac{E q(n \tau)}{\alpha^{n}+\sqrt{\tau} g^{n}}+\lambda\left(\alpha^{n}+\sqrt{\tau} g^{n}\right)^{2}=0 .
$$

This implicit equation is solved by a Newton method with initial data $g^{n-1}$. 
In fact, equation (1.8) may admit several solutions and the convergence of Newton iterations is correlated to the convexity of $\phi_{n}$. The function $\phi_{n}$ is concave on -$] \frac{\alpha^{n}}{\sqrt{\tau}}, \xi^{n}[$ and convex on $]-\xi^{n},+\infty\left[\right.$, where $\xi^{n}$ is the unique real solution of $P_{n}\left(\alpha+\sqrt{\tau} \xi^{n}\right)=0$, with

$$
P_{n}(x)=x-2 E q^{n}+2 \lambda x^{3} .
$$

Therefore, equation (1.8) admits at most three solutions and a unique solution if $E q^{n} \geq \sqrt{\tau} / 3$. Let us point out that we have here an almost exact computation - up to the Newton iterations - of the solution of the semi-discrete problem. In particular, no truncation of the domain is involved.

In the sequel, we will be interested by the more general problem:

$$
\left\{\begin{array}{l}
\frac{u^{n+1}-u^{n}}{\tau}-u_{x x}^{n+1}=0, x>0 \\
u_{x}^{n+1}(0)=-\log u^{n+1}(0)-\frac{E q^{n+1}}{u^{n+1}(0)}+\lambda u^{n+1}(0)^{2} \\
u^{0}=u_{0} .
\end{array}\right.
$$

The main property of the numerical scheme described in equations (1.7) and (1.9) lies in its order preservation corresponding to the maximum principle stated for (1.1):

Theorem 1.1. Let $u_{1}^{n}, u_{2}^{n}$ be the solutions of (1.9) with

$$
E q=E_{1} q_{1} \leq E_{2} q_{2}, u_{0}=u_{01} \leq u_{02}, \lambda=\lambda_{1} \geq \lambda_{2} .
$$

Then, as long as $u_{2}^{p}(0)>\sqrt{8 \tau}$ for all integer $p \in[1, n]$, we have $u_{1}^{n} \leq u_{2}^{n}$.

Proof. Let us assume $w^{n}=u_{1}^{n}-u_{2}^{n} \leq 0$ and $u_{2}^{n+1}(0)>\sqrt{8 \tau}$.

Let $\left(w^{n+1}\right)_{+}=\max \left(w^{n+1}, 0\right)$ and $\omega^{n}=\operatorname{Supp}\left(\left(w^{n+1}\right)_{+}\right)$. The equation (1.9) implies:

$$
\left(w^{n+1}-w^{n}\right)-\tau w_{x x}^{n+1}=2 \tau \delta_{x=0}\left(f\left(u_{1}^{n+1}\right)-f\left(u_{2}^{n+1}\right)\right),
$$

with $f(u)=\log u+\frac{E q}{u}-\lambda u^{2}$. Multiplying (1.10) by $w_{+}^{n+1}$ and integrating, we get:

$$
\left\|w_{+}^{n+1}\right\|_{L^{2}}^{2}-\int_{\omega^{n}} w^{n} w_{+}^{n+1}-\tau \int_{\omega^{n}} w_{x x}^{n+1} w_{+}^{n+1}=2 \tau\left(f\left(u_{1}^{n+1}(0)\right)-f\left(u_{2}^{n+1}(0)\right)\right) w_{+}^{n+1}(0)
$$

so that, by integration by parts:

$$
\begin{aligned}
\left\|w_{+}^{n+1}\right\|_{L^{2}}^{2}+\int_{\omega^{n}}\left(w_{+}^{n+1}-w^{n}\right)^{2}-\int_{\omega^{n}}\left(w^{n}\right)^{2}+2 \tau \int_{\omega^{n}}\left(\left(w_{+}^{n+1}\right)_{x}\right)^{2}= \\
4 \tau\left(f\left(u_{1}^{n+1}(0)\right)-f\left(u_{2}^{n+1}(0)\right)\right) w_{+}^{n+1}(0) \\
\leq 4 \tau\left(\log u_{2}^{n+1}(0)-\log u_{1}^{n+1}(0)\right) w_{+}^{n+1}(0) \leq \frac{4 \tau}{u_{2}^{n+1}(0)}\left(w_{+}^{n+1}(0)\right)^{2} .
\end{aligned}
$$


On the other hand:

$$
\begin{aligned}
\frac{4 \tau}{u_{2}^{n+1}(0)}\left(w_{+}^{n+1}(0)\right)^{2} & =-\frac{4 \tau}{u_{2}^{n+1}(0)} \int_{0}^{+\infty} \partial_{x}\left(\left(w_{+}^{n+1}\right)^{2}\right) \mathrm{d} x \\
& =-\frac{4 \tau}{u_{2}^{n+1}(0)} \int_{0}^{+\infty} 2 w_{+}^{n+1}\left(w_{+}^{n+1}\right)_{x} \mathrm{~d} x \\
& \leq \frac{8 \tau}{\left(u_{2}^{n+1}(0)\right)^{2}}\left\|w_{+}^{n+1}\right\|_{L^{2}}^{2}+2 \tau\left\|\left(w_{x}^{n+1}\right)_{+}\right\|_{L^{2}}^{2}
\end{aligned}
$$

Hence,

$$
\left(1-\frac{8 \tau}{\left(u_{2}^{n+1}(0)\right)^{2}}\right)\left\|w_{+}^{n+1}\right\|_{L^{2}}^{2}+\int_{\omega^{n}}\left(w_{+}^{n+1}-w^{n}\right)^{2} \mathrm{~d} x \leq \int_{\omega^{n}}\left(w^{n}\right)^{2} \mathrm{~d} x
$$

and, since $u_{2}^{n+1}(0)>\sqrt{8 \tau}, w_{+}^{n+1}=0$, so that $u_{1}^{n+1} \leq u_{2}^{n+1}$.

Therefore, as in the continuous case, we have a comparison principle, except when $R^{n}$ is too small, and that is why we introduce the following definition:

Definition 1.2. The flame quenches numerically if there exists some time $n \tau$ such that $R^{n} \leq \sqrt{8 \tau}$, for any positive $n$.

The initiation of the flame with such a definition is a consequence of the:

Proposition 1.3. Under the asymptotic condition $E q(t)=O\left(t^{1 / 2-\varepsilon}\right)$, for any positive $\varepsilon, g^{1} \sim \frac{\sqrt{E q(\tau)}}{\tau^{1 / 4}}$ as $\tau$ tends to 0 .

Such a result implies $R^{1} \sim \sqrt{E q(\tau)} \tau^{1 / 4}$ and the condition $R^{1}>\sqrt{8 \tau}$ is verified for $\tau$ small.

Proof. Given $c \geq 0$, let $\phi_{1}$ defined as in (1.8). We have:

$$
\phi_{1}\left(\frac{c \sqrt{E q(\tau)}}{\tau^{1 / 4}}\right)=\left(c-\frac{1}{c}\right) \frac{\sqrt{E q(\tau)}}{\tau^{1 / 4}}-\log \left(\tau^{1 / 4} c \sqrt{E q(\tau)}\right)+\lambda \tau^{1 / 2} c^{2} E q(\tau)
$$

For $\tau$ small enough, and $c \neq 1$,

$$
\phi_{1}\left(\frac{c \sqrt{E q(\tau)}}{\tau^{1 / 4}}\right)=\left(c-\frac{1}{c}\right) \frac{\sqrt{E q(\tau)}}{\tau^{1 / 4}}+o\left(\tau^{1 / 4}\right)
$$

and we infer from this the expected equivalent of $g^{1}$.

\section{Convergence of the scheme}

Let us set $e^{n}=u^{n}-u\left(t^{n},.\right)$, where $t^{n}=n \tau$. Then, $\left(e^{n}\right)_{n}$ is solution of the sequence of elliptic problems:

$$
\left\{\begin{array}{l}
e_{x x}^{n+1}-\frac{e^{n+1}}{\tau}=-\frac{e^{n}}{\tau}-\frac{1}{\tau}\left(u\left(t^{n+1}\right)-u\left(t^{n}\right)-\tau u_{t}\left(t^{n+1}\right)\right), \quad x>0 \\
e_{x}^{n+1}(0)=f^{n+1}\left(u^{n+1}(0)\right)-f^{n+1}\left(u\left(t^{n+1}, 0\right)\right):=\beta^{n+1} e^{n+1}(0) \\
e^{0}=0
\end{array}\right.
$$

where $\beta^{n+1}$ depends on $u\left(t^{n+1}, 0\right)$ and $e^{n+1}(0)$. 
Our goal here is to compare (2.1) with the heat equation with Robin condition, namely:

$$
\left\{\begin{array}{l}
-e_{x x}^{n+1}+\frac{e^{n+1}}{\tau}=\frac{e^{n}}{\tau}+g^{n+1}, \quad x>0 \\
e_{x}^{n+1}(0)=\chi^{n+1} e^{n+1}(0), \\
e^{0}=0
\end{array}\right.
$$

where $\chi^{n+1}$ is the linearization of $\beta^{n+1}$ when $e^{n+1}(0)$ is small in comparison with $u\left(t^{n+1}, 0\right)$ :

$$
\chi^{n+1}=\frac{E q^{n+1}}{u\left(t^{n+1}, 0\right)^{2}}-\frac{1}{u\left(t^{n+1}, 0\right)}+2 \lambda u\left(t^{n+1}, 0\right),
$$

and where $g^{n+1}$ is defined by:

$$
g^{n+1}=\frac{1}{\tau}\left(u\left(t^{n+1}\right)-u\left(t^{n}\right)-\tau u_{t}\left(t^{n+1}\right)\right) .
$$

Let us begin with the study of (2.2):

Lemma 2.1. Let $e^{n}$, solution of (2.2), such that $\left|e^{n}\right| \rightarrow 0$ as $|x| \rightarrow+\infty$. Let us assume that the quantity $\left\|g^{n}\right\|_{\infty}$ is finite.

(i) If $\chi^{n+1} \geq 0,\left\|e^{n+1}\right\|_{\infty} \leq\left\|e^{n}\right\|_{\infty}+\tau\left\|g^{n+1}\right\|_{\infty}$.

(ii) If $\chi^{n+1}<0$, let us define $\chi=\min _{t \in\left[t^{1}, t^{n+1}\right]}\left(\frac{E q(t)}{u(t, 0)^{2}}-\frac{1}{u(t, 0)}+2 \lambda u(t, 0)\right)$, and $x^{n}=\left\|e^{-\chi x /(1+x)} e^{n+1}\right\|_{\infty}$. Then, when $\tau$ is sufficiently small so that $1-2 \tau \chi^{2}>0$,

$$
x^{n+1} \leq \frac{x^{n}}{1-2 \tau \chi^{2}}+\frac{\tau e^{-\chi}\left\|g^{n+1}\right\|_{\infty}}{1-2 \tau \chi^{2}} .
$$

Proof. First, assume that $\chi^{n+1}>0$. Since $e^{n+1} \rightarrow 0$ as $|x| \rightarrow+\infty$, if $\max e^{n+1}>0$, this maximum is achieved in $x_{0}>0$, because of the Robin condition, and:

$$
e^{n+1}(x) \leq e^{n+1}\left(x_{0}\right) \leq e^{n}\left(x_{0}\right)+\tau g^{n+1}\left(x_{0}\right) .
$$

Similarly, considering a negative minimum $x_{0}$, we get:

$$
e^{n+1}(x) \geq e^{n+1}\left(x_{0}\right) \geq e^{n}\left(x_{0}\right)+\tau g^{n+1}\left(x_{0}\right),
$$

and estimations are the same as in the case $\chi^{n+1}=0$.

Let us now turn to the case $\chi<0$. For any $\chi \in \mathbb{R}$, we consider $w^{n+1}=e^{-\chi x /(1+x)} e^{n+1}$. Then:

$$
\left\{\begin{array}{l}
-w_{x x}^{n+1}-\frac{2 \chi}{(1+x)^{2}} w_{x}^{n+1}+\left(\frac{1}{\tau}+\frac{2 \chi}{(1+x)^{3}}-\frac{\chi^{2}}{(1+x)^{4}}\right) w^{n+1}=\frac{1}{\tau} w^{n}+e^{-\chi x /(1+x)} g^{n+1} \\
w_{x}^{n+1}(0)=0
\end{array}\right.
$$

As before, if $x^{n}=\left\|e^{-\chi x /(1+x)} e^{n+1}\right\|_{\infty}$, when $\tau$ is sufficiently small,

$$
x^{n+1} \leq \frac{x^{n}}{1-2 \tau \chi^{2}}+\frac{\tau\left\|e^{-\chi} g^{n+1}\right\|_{\infty}}{1-2 \tau \chi^{2}} .
$$

This lemma will apply when $\left(g^{n+1}\right)$ is bounded and when we have a lower bound on $\left(\beta^{n+1}\right)$, and it will give us the following result of convergence: 
Proposition 2.2. Let $\varepsilon>0$ be small enough. There exist $C_{1}, C_{2}, C_{3}>0$ such that the following estimates hold:

(i) During the initiation phase, on which the equivalent of the radius $R(t) \sim R_{0} t^{1 / 4}$, obtained in [1], is valid, we have:

$$
\left|e^{n}\right| \leq\left|e^{1}\right|+C_{1} \tau^{1 / 4}
$$

(ii) Otherwise, as long as $u\left(t^{n+1}, 0\right) \geq \varepsilon$,

$$
\left|e^{n}\right| \leq C_{3} e^{\left(\frac{1}{\varepsilon} \log \varepsilon\right)^{2}}\left(\left|e^{1}\right|+C_{1} \tau^{1 / 4}+C_{2} \tau+\tau \varphi(\varepsilon)\right),
$$

where $\varphi$ is a decreasing function such that $\varphi(\varepsilon) \sim|\log \varepsilon|$.

Assume Proposition 2.2 is true for the $n$ first iterations. Let us prove it at the $(n+1)^{\text {th }}$ iteration; it will follow from the following lemmas:

Lemma 2.3. Let $\varepsilon>0$, and $\left(\beta^{n}\right)_{n}$ defined in (2.1). If $u\left(t^{n}, 0\right) \geq \varepsilon$,

$$
\beta^{n+1} \geq \min \left(\frac{-1}{\varepsilon}, \frac{1}{\varepsilon} \log \frac{\varepsilon}{2\|u\|_{\infty}}\right)
$$

Proof. In our problem,

$$
\beta^{n+1}=-\frac{1}{e^{n+1}(0)} \log \left(1+\frac{e^{n+1}(0)}{u\left(t^{n+1}, 0\right)}\right)+\frac{E q^{n+1}}{u\left(t^{n+1}, 0\right)\left(u\left(t^{n+1}, 0\right)+e^{n+1}(0)\right)}+\lambda\left(2 u\left(t^{n+1}, 0\right)+v^{n+1}(0)\right) .
$$

We want a lower bound on $\left(\beta^{n}\right)$, depending on $\varepsilon$ : let us first notice that, as long as $u\left(t^{n+1}, 0\right) \geq \varepsilon$, the result of convergence on the previous step implies $u^{n}(0) \geq 3 \varepsilon / 4$. Since

$$
u^{n+1}(0)-u^{n}(0)=\sqrt{\tau} f^{n+1}\left(u^{n+1}(0)\right)-\tau \int_{\mathbb{R}} \frac{4 \pi \xi^{2}}{1+4 \pi \xi^{2} \tau} \hat{u}^{n}(\xi) \mathrm{d} \xi,
$$

with $f^{n}$ denoting the function defined in the first section, we get $u^{n+1}(0) \geq \varepsilon / 2$ for $\tau$ small enough. Then, if $v^{n+1}(0)>0$ and if we look at the times $t^{n+1}$ such that $u\left(t^{n+1}, 0\right) \geq \varepsilon$,

$$
\beta^{n+1} \geq \frac{-1}{e^{n+1}(0)} \log \left(1+\frac{e^{n+1}(0)}{u\left(t^{n+1}, 0\right)}\right) \geq-\frac{1}{\varepsilon}
$$

If $e^{n+1}(0)<0$ and if $u\left(t^{n+1}, 0\right) \geq \varepsilon$, there holds $u^{n+1}(0) \geq \varepsilon / 2$ and $\beta^{n+1} \geq \frac{1}{\varepsilon} \log \frac{\varepsilon}{2\|u\|_{\infty}}$. It remains to check that $\beta^{n}$ is not too small during the initiation of the flame: because of the term $E q^{n+1}$ which does not vanish at the beginning, $\beta^{n+1}>0$ in the first times for $\varepsilon$ small enough. On the contrary, $\beta^{n+1} \rightarrow-\infty$ during the quenching phase if we assume that the function $q$ is compactly supported, hence the interest to suppose $u\left(t^{n}, 0\right) \geq \varepsilon>0$.

Lemma 2.4. (i) During the initiation phase,

$$
\left\|g^{n}\right\|_{\infty} \leq \frac{C \tau}{(n \tau)^{7 / 4}}
$$

(ii) Otherwise, as long as $u\left(t^{n}, 0\right) \geq \varepsilon$,

where $\varphi(\varepsilon) \sim|\log \varepsilon|$ as $\varepsilon$ tends to 0 .

$$
\left\|g^{n}\right\|_{\infty} \leq C \tau \varphi(\varepsilon)
$$


Proof. The quantity $g^{n}$ is defined by:

$$
\left.g^{n}=\frac{1}{\tau}\left(u\left(t^{n+1}\right)-u\left(t^{n}\right)-\tau u_{t}\left(t^{n+1}\right)\right)=\tau u_{t t}(t), t \in\right] t^{n}, t^{n+1}[.
$$

The point $(i)$ is a direct consequence of the ansatz $R(t) \sim R_{0} t^{1 / 4}[1]$ and $u_{t t}(t, 0) \sim C \tau^{-7 / 4}$.

If we except the initiation and quenching phases, the quantity $\left(g^{n}\right)_{n}$ is bounded, and we have to obtain a uniform estimate, depending on $\varepsilon$, of $\left\|u_{t t}\right\|_{\infty}$, since the problem is no longer Lipschitz in the quenching phase. Let us denote by $t_{0}=n_{0} \tau$ the time such that $\left(0, t_{0}\right)$ corresponds to the initiation phase. We assume that $u(t, 0) \geq \varepsilon$ for any $t$ greater than $t_{0}$. Let $\beta=\inf \beta^{n}$, and $0<\gamma<1 / 2$. In the following, $\|\cdot\|_{H^{\alpha, \delta}(] t_{1}, t[\times \mathbb{R})}$ denotes the norm in the corresponding Hölder spaces for any $\alpha, \delta$ nonintegral numbers; $\langle\cdot\rangle_{] t_{1}, t}^{(\theta)}$ is the hölderian semi-norm of order $\theta$, with $0<\theta<1$. We have:

$$
\left\{\begin{array}{l}
u_{t}-u_{x x}=0, \quad x>0, \\
u_{x}(t, 0)=-\log u(t, 0)+\frac{E q(t)}{u(t, 0)}+\lambda u(t, 0)^{2}=f(u(t, 0)), \\
u(0, .)=0 .
\end{array}\right.
$$

Looking at times such that $u\left(t^{n}, 0\right) \geq \varepsilon$, by parabolic regularity [9], there holds:

$$
\begin{aligned}
\left\|u_{t t}\right\|_{L^{\infty}(] t_{0}, t[\times \mathbb{R})} & \leq\|u\|_{H^{\gamma / 2+2, \gamma+4}(] t_{1}, t[\times \mathbb{R})} \\
& \leq C\|u(., 0)\|_{H^{\gamma / 2+1}(] t_{1}, t[\times \mathbb{R})} \leq C\|u\|_{H^{\gamma / 2+1, \gamma+2}(] t_{1}, t[\times \mathbb{R})} .
\end{aligned}
$$

Yet, $\|u\|_{H}^{\gamma / 2+1, \gamma+2}(] t_{1}, t[\times \mathbb{R})$ is controlled (see [9]) by $\|u(., 0)\|$, and expanding it, we have a term in $\varphi(\varepsilon)$, corresponding to the non linear singularity: $\varphi(\varepsilon) \leq C|\log \varepsilon|$, and when the function $q$ is compactly supported, $\varphi(\varepsilon) \sim|\log \varepsilon|$, since we are looking at the quenching of the flame. Thus,

$$
\begin{aligned}
\left\|u_{t t}\right\|_{L^{\infty}(] t_{1}, t[\times \mathbb{R})} & \leq C\left(\varphi(\varepsilon)+\langle u(., 0)\rangle_{] t_{1}, t[}^{(\gamma)}+\langle u(., 0)\rangle_{] t_{1}, t\right]}^{(2 \gamma)}\right) \\
& \leq C\left(\varphi(\varepsilon)+\tau^{1-2 \gamma}\left\|u_{t}(., 0)\right\|_{L^{\infty}(] t_{1}, t[)}\right) \\
& \leq C\left(\varphi(\varepsilon)+\tau^{1-2 \gamma} \varphi(\varepsilon)\|u\|_{L^{\infty}(] t_{1}, t[\times \mathbb{R})}\right) \leq C \varphi(\varepsilon)
\end{aligned}
$$

hence the desired estimate on $\left\|g^{n}\right\|_{\infty}$ during the development of the flame.

Proof of Proposition 2.2. The point (i) ensues directly from Lemma 2.1 and Lemma 2.4, applied on the initiation phase $\left(0, t_{0}\right)$.

For the point (ii), we firstly look at the times $n \tau>t_{0}$ such that $\beta^{n} \geq 0$. In fact, we are dealing here with a classical nonlinear Lipschitz problem, and we get:

$$
\left|e^{n}\right| \leq\left|e^{1}\right|+C_{1} \tau^{1 / 4}+C_{2} \tau .
$$

As soon as $\beta^{n} \leq 0$, we apply the previous lemmas, and since $O(1)$ times scales are considered here, if $\beta=\inf \beta^{n}$, we get:

$$
x^{n} \leq e^{\beta^{2}} x^{n_{1}}+e^{\beta^{2}} \tau \sum_{k=1}^{n}\left\|e^{-\beta} g^{n-k+1+n_{1}}\right\|_{\infty} \leq e^{\beta^{2}} x^{n_{1}}+C e^{\beta^{2}} \tau \varphi(\varepsilon),
$$

where $x^{n}=\left\|e^{-\chi x /(1+x)} u\right\|_{\infty}$. Hence,

$$
\left|e^{n}\right| \leq C e^{\beta^{2}}\left(\left|e^{n_{1}}\right|+\tau \varphi(\varepsilon)\right) .
$$


Thus, $\varepsilon$ being fixed, we can choose $\tau$ sufficiently small to control the error done with this numerical scheme, expressed by the estimates (2.4) and (2.5).

Remark. we may improve our convergence result at low cost by choosing $\varepsilon=\sqrt{1 /|\log \tau|}$. Theorem 2.2 still holds as the number of iterations tends to $+\infty$. We will denote by $n_{\max }(\tau)$ the iteration $n$ such that $R^{n}>\sqrt{1 /|\log \tau|}$ and $R^{n+1} \leq \sqrt{1 /|\log \tau|}$. If the flame quenches in a finite time $t_{\max }$,

$$
t_{\max }=n_{\max }(\tau) \tau+O\left(\frac{1}{|\log \tau|}\right) .
$$

This provides from the convergence result presented above and the equivalent (see [1]):

$$
R(t) \sim \frac{\sqrt{\pi}}{2} \sqrt{t_{\max }-t} \text { as } t \rightarrow t_{\max }
$$

\section{Asymptotic Behaviour of the scheme}

As seen previously, we have convergence of the scheme for finite times as the number of iterations tends to $+\infty$. More important for the validation of numerical simulations for such models is the similarity of the qualitative asymptotic behaviour of $R^{n}$ for large $n$ (particularly when $q^{n}=0$ ) with that of the continuous case, i.e. extinction or stabilization towards a critical radius.

First, let us recall the results proved in the study of the large-time properties of (1.1). Critical radiuses are the solutions of the equation:

$$
\log R=\lambda R^{2}
$$

depending on the parameter $\lambda$. There exists a critical value $\lambda_{\text {cr }}$ of $\lambda$ such that:

- for $\lambda>\lambda_{\mathrm{cr}}$, there is no solution,

- for $\lambda<\lambda_{\text {cr }},(3.1)$ admits two solutions $R_{1}<R_{2}$.

For $\lambda>\lambda_{\text {cr }}$, the flame always quenches. For $\lambda<\lambda_{\text {cr }}$, the asymptotic behaviour depends on the energy input parameter $E$ : the flame radius either tends to 0 - finite or infinite time quenching cases -, or to $R_{1}$ or $R_{2}-$ stabilization cases -, according to whether, given $q$, the parameter $E$ is below, equal to, or above a critical parameter $E_{\mathrm{cr}}(q)$.

The results obtained for the numerical scheme are almost the same, up to a small error in $\tau$ :

Theorem 3.1. Assume $\lambda>\lambda_{\mathrm{cr}}$. Then, the numerical solution quenches. If the continuous solution quenches in finite time $t_{\max }$, we have the estimate (2.7) about $t_{\max }$.

Theorem 3.2. For any positive $\tau$, there exists $\lambda_{\mathrm{cr}}(\tau) \leq \lambda_{\mathrm{cr}}$ such that, for $\lambda<\lambda_{\mathrm{cr}}(\tau)$, there exists $E_{\mathrm{cr}}(q, \tau)>0$ such that:

- if $E<E_{\mathrm{cr}}(q, \tau)$, the solution quenches after $n_{\max }(\tau)$ iterations,

- if $E>E_{\mathrm{cr}}(q, \tau), R^{n} \rightarrow R_{2}$,

- if $E=E_{\mathrm{cr}}(q, \tau), R^{n} \rightarrow R_{1}$.

Moreover,

$$
\lim _{\tau \rightarrow 0} \lambda_{\mathrm{cr}}(\tau)=\lambda_{\mathrm{cr}}, \text { and } \lim _{\tau \rightarrow 0} E_{\mathrm{cr}}(q, \tau)=E_{\mathrm{cr}}(q) .
$$

If the solution of the continuous problem quenches in finite time $t_{\max }$, the estimate (2.7) about $t_{\max }$ also holds.

The difference observed for the critical value of $\lambda$ is due to the fact that the scheme under consideration carries an extra (numerical) lot of diffusion.

The remaining part of this section is devoted to the proof of Theorems 3.1 and 3.2. Let us start it by recalling the classical sub and super solutions results: if $f$ is a locally Lipschitz, real-valued function, we shall say that 
$\underline{u}($ resp. $\bar{u})$ is a subsolution (resp. supersolution) of

$$
-u^{\prime \prime}=\delta_{x=0} f(u)
$$

if and only if there holds, in the $H^{-1}$ sense:

$$
-\underline{u}^{\prime \prime} \leq \delta_{x=0} f(\underline{u})\left(\text { resp. }-\bar{u}^{\prime \prime} \geq \delta_{x=0} f(\bar{u})\right) .
$$

Let us consider the implicit semi-discrete approximation problem of the Cauchy problem

$$
\begin{gathered}
u_{t}-u_{x x}=2 \delta_{x=0} f(u(t, 0)), u(0, .)=0: \\
u_{x x}^{n+1}-\frac{u^{n+1}}{\tau}=-\frac{u^{n}}{\tau}-2 \delta_{x=0} f\left(u^{n+1}\right), u^{0}=0 .
\end{gathered}
$$

Theorem 3.3. ( $i$ ) Assume the existence of a subsolution $\underline{u}$ to problem (3.2). Then, the solution ( $\left.u^{n}\right)$ of problem (3.3), with $u^{0}=\underline{u}$, is time-increasing on its life set. Moreover, if this set is infinite and $u^{n} \in L^{\infty}(\mathbb{N} \times \mathbb{R})$, then the sequence $u^{n}$ is uniformly convergent on every compact of $\mathbb{R}$ towards the minimal solution $u_{\infty}$ of (3.2) such that $u_{\infty} \geq \underline{u}$.

(ii) Assume the existence of a supersolution $\bar{u}$ to problem (3.2). Then, the solution $\left(u^{n}\right)$ of problem (3.3), with $u^{0}=\bar{u}$, is time-decreasing on its life set. Moreover, if this set is infinite and $u^{n} \in L^{\infty}(\mathbb{N} \times \mathbb{R})$, then the sequence $u^{n}$ is uniformly convergent on every compact of $\mathbb{R}$ towards the maximal solution $u_{\infty}$ of (3.2) such that $u_{\infty} \leq \bar{u}$.

Before investigating this asymptotic behaviour, let us give a result, valid for any $\lambda>0$.

Proposition 3.4. There exists $C$ positive such that $u^{n} \leq C$ on the life set of $\left(u^{n}\right)$ solution of (1.9).

Proof. Let us consider $C>0$ such that $u^{n} \leq C$ and

$$
0 \leq E<E_{0}=\frac{1}{\|q\|_{\infty}}\left(\lambda C^{3}-C \log C\right)
$$

Then $v^{n}=u^{n}-C$ is such that

$$
v_{x x}^{n+1}-\frac{v^{n+1}}{\tau}=-\frac{v^{n}}{\tau} \geq 0
$$

and by the maximum principle,

$$
\max _{\mathbb{R}_{+}} v^{n+1} \leq\left(v^{n+1}(0)\right)^{+} .
$$

If $v^{n+1}(0)>0$, the Hopf lemma implies $v_{x}^{n+1}(0)=u_{x}^{n+1}(0)<0$, in contradiction with

$$
u_{x}^{n+1}(0) \geq \frac{1}{u^{n+1}(0)}\left(\lambda C^{3}-C \log C-E q\right) \geq 0 .
$$

So, $u^{n+1} \leq C$.

In the sequel, $u_{E}^{n}$ will denote the solution of (1.4), and $R_{E}^{n}=u_{E}^{n}(0)$ will be the approximated flame radius. 


\subsection{Asymptotic behaviour when $\lambda>\lambda_{\text {cr }}$}

Proof of Theorem 3.1. Let $n_{0} \in \mathbb{N}, \forall n \geq n_{0}, q^{n} \leq \frac{\varepsilon}{E_{0}}$, where $E_{0}>0$ is chosen such that:

$$
\log C+\frac{\varepsilon}{C}-\lambda C^{2} \leq 0
$$

$C>0$ being the constant defined in the proof of the previous proposition. As long as $u_{E}^{n}(0)>\sqrt{8 \tau}$, we have $u_{E}^{n+n_{0}} \leq v^{n}$, where $v^{n}$ is solution of:

$$
\left\{\begin{array}{l}
v_{x x}^{n+1}-\frac{v^{n+1}}{\tau}=-\frac{v^{n}}{\tau}, x>0 \\
v_{x}^{n+1}(0)=-\log v^{n+1}(0)-\frac{\varepsilon}{v^{n+1}(0)}+\lambda v^{n+1}(0)^{2} \\
v^{0}=C
\end{array}\right.
$$

The constant $C$ being a supersolution to (3.2) with $f(u):=2\left(\log u+\frac{\varepsilon}{u}-\lambda u^{2}\right)$, Theorem 3.3 applies. The function $f_{\varepsilon}$ has a unique zero $u_{\varepsilon, \infty}$ with $\lim _{\varepsilon \rightarrow 0} u_{\varepsilon, \infty}=0$, and $u_{\varepsilon, \infty}$ is the only non-negative solution of (3.2) with $f=f_{\varepsilon}$. Consequently, $v^{n}$ converges locally towards $u_{\varepsilon, \infty}$. Since $\varepsilon$ can be arbitrarily small, there exists $n \in \mathbb{N}^{*}$ such that $u_{E}^{n}(0) \leq \sqrt{8 \tau}$, i.e. the flame quenches.

\subsection{Asymptotic behaviour when $\lambda<\lambda_{\mathrm{cr}}$ : $\boldsymbol{E}$ small or large}

Theorem 3.3 leads us directly to the fact that the solution quenches for small energies:

Proposition 3.5. There exists $E_{0}>0$ such that, for all $E \leq E_{0}, u_{E}^{n}$ quenches.

Proof. Set $C=1 / 2$. Let $E \leq \frac{\lambda C^{3}-C \log C}{\|q\|_{\infty}}=E_{0}$. Then, $u^{n} \leq 1 / 2$, as in the proof of Proposition 3.4. The comparison principle and the asymptotic results written in Theorem 3.3 imply that there exists $n \in \mathbb{N}^{*}$ such that $u_{E}^{n}(0) \leq \sqrt{8 \tau}$.

We turn now to the stabilization case. In order to do so, let us introduce a family of subsolutions to (3.2), presented in [1] and [12] for the continuous case. Define $\phi_{b}(x)$ by:

$$
\phi_{b}(x)=-\left(\log b-\lambda b^{2}\right)|x|+b, R_{1}<b<R_{2}
$$

$\underline{\phi}_{b}=\max \left(\phi_{b}, 0\right)$ is a subsolution to $(3.2)$ with $f(u)=2\left(\log u+\frac{E q}{u}-\lambda u^{2}\right)$. This family of subsolutions leads us to the following:

Proposition 3.6. There exists $E_{1}>0$ such that $\forall E>E_{1}$, and for all small enough $\tau, \lim _{n \rightarrow+\infty} u_{E}^{n}(0)=R_{2}$.

Proof. It is proved in [1] and [12] that, for $E_{1}$ sufficiently large, there exists $t>0$ such that:

$$
\underline{\phi}_{\left(R_{2}+R_{\mathrm{cr}}\right) / 2}(x) \leq u_{E_{1}}(t, x) \forall x \in \mathbb{R} .
$$

Therefore, by Proposition 2.2, $\tau$ being definitely chosen, there exists $n_{0}$ positive such that:

$$
\underline{\phi}_{R_{\mathrm{cr}}}=\underline{\phi}(x) \leq u_{E_{1}}^{n_{0}}(x)
$$


The comparison principle implies $u_{E_{1}}^{n+n_{0}} \geq v^{n}$, where

$$
\left\{\begin{array}{l}
v_{x x}^{n+1}-\frac{v^{n+1}}{\tau}=-\frac{v^{n}}{\tau}, x>0 \\
v_{x}^{n+1}(0)=-\log v^{n+1}(0)-\frac{E_{1} q^{n+1}}{v^{n+1}(0)}+\lambda v^{n+1}(0)^{2} \\
v^{0}=\underline{\phi}
\end{array}\right.
$$

as long as $u_{E_{1}}^{n}(0) \geq \sqrt{8 \tau}$. The solution of (3.5) is time-increasing and bounded, and $v^{n}$ converges towards the smaller solution, higher than $\underline{\phi}$, of the equation:

$$
-u^{\prime \prime}=2 \delta_{x=0}\left(\log u-\lambda u^{2}\right),
$$

solution equal to $R_{2}$. Hence, $\liminf _{n \rightarrow+\infty} u_{E_{1}}^{n} \geq R_{2}$. And since $u_{E}>u_{E_{1}}$ for all $E>E_{1}$, there holds $\liminf _{n \rightarrow+\infty} u_{E}^{n}=R_{2}$.

On the other hand, as in the proof of Proposition 3.1, $u_{E}^{n} \leq v^{n}$ where $v^{n}$ tends to the largest zero of:

$$
u \mapsto u \log u+\varepsilon-\lambda u^{3}
$$

zero arbitrarily near to $R_{2}$ for $E>E_{1}$. Hence, $\limsup _{n \rightarrow+\infty} u_{E}^{n} \leq R_{2}$.

\subsection{Large-time behaviour when $\lambda<\lambda_{\mathrm{cr}}$ : Existence and uniqueness of the threshold}

We have just seen that the numerical solution quenches or stabilizes to $R_{2}$, depending on the value of the energy input parameter $E$. Let us introduce the sets corresponding to these situations:

$$
\begin{gathered}
X_{+}=\left\{E>0, \lim _{n \rightarrow+\infty} R_{E}^{n}=R_{2}\right\} \\
X_{-}=\left\{E>0, \exists n>0, R_{E}^{n}(0) \leq \sqrt{8 \tau}\right\} .
\end{gathered}
$$

Proposition 3.7. $X_{+}$and $X_{-}$are open subsets of $\mathbb{R}_{+}^{*}$.

This proposition implies, by connectedness of $\mathbb{R}_{+}^{*}$, that the set $X_{0}=\mathbb{R}_{+}^{*} \backslash\left(X_{+} \cup X_{-}\right)$is non empty. Besides, by Theorem 1.1, $X_{0}$ is a closed interval, when not reduced to one point.

As in the study of long-time behaviour of (1.1), the main tool of the proof of this proposition is the following result:

Theorem 3.8. Let $E>0$. For all $n \in \mathbb{N}$, the function $x \mapsto u_{E}^{n}(x)-R_{1}$ admits at most two zeroes, as long as $u^{n}(0)>\sqrt{8 \tau}$.

Proof. Let us consider the sequence $\left(v^{n}\right)$ and the elliptic operator $L$, defined by:

$$
v^{n}:=u^{n}-R_{1},
$$

and

$$
\left\{\begin{array}{l}
L v^{n+1}=v_{x x}^{n+1}-\frac{v^{n+1}}{\tau}=-\frac{v^{n}}{\tau}, \quad x>0 \\
v_{x}^{n+1}(0)=-\log u^{n+1}(0)-\frac{E q^{n+1}}{u^{n+1}(0)}+\lambda\left(u^{n+1}(0)\right)^{2} \\
u^{0}=0 .
\end{array}\right.
$$

Since $u_{E}^{n}$ is even, the zeros of $v^{n}$ are symmetrical: therefore, in the forthcoming, we will only look at the zeros of $v^{n}$ on $\mathbb{R}_{+}$. Assume Theorem 3.8 to be true up to the $n^{\text {th }}$ iteration. Then, either $v^{n}$ has a unique simple zero in $x_{0}>0$, or $v^{n}$ has a simple or double zero in $x_{0}=0$, or $v^{n}$ does not vanish on $\mathbb{R}_{+}$. 
Case 1: $v^{n}<0$.

Then $L v^{n+1}>0$, and

$$
\max _{\mathbb{R}_{+}} v^{n+1} \leq \max \left(v^{n+1}(0), 0\right) .
$$

If there exists a local non negative maximum achieved in $x_{1} \in \mathbb{R}_{+}^{*}, v_{x}^{n+1}\left(x_{1}\right)=0$ and

$$
0 \geq v_{x x}^{n+1}\left(x_{1}\right)=\frac{\left(v^{n+1}-v^{n}\right)\left(x_{1}\right)}{\tau}>\frac{v^{n+1}\left(x_{1}\right)}{\tau},
$$

in contradiction with $v^{n+1}\left(x_{1}\right) \geq 0$, which ends the case $v^{n+1}(0)<0$. If $v^{n+1}(0)>0$, since $v^{n+1} \rightarrow-R_{1}$ as $x \rightarrow+\infty, v^{n+1}$ has a unique zero at $x_{1}>0$, and this zero is simple, but otherwise $v_{x x}^{n+1}\left(x_{1}\right)>0$ and $v_{x}^{n+1}$ is positive in a vicinity of $x_{1}$. If $v^{n+1}(0)=0, v_{x}^{n+1}(0)=-\frac{E q^{n+1}}{u^{n+1}(0)} \leq 0$, and $v_{x x}^{n+1}(0)=-v^{n}(0)$ : we can only have a simple or a double zero in 0 .

Case 2: $v^{n}$ has a simple zero in $x_{0}>0$.

If we have a non negative maximum of $v^{n+1}$ achieved in some $x_{1}>x_{0}$, then $v_{x x}^{n+1}\left(x_{1}\right) \leq 0$; but $v_{x x}^{n+1}\left(x_{1}\right) \geq$ $-\frac{v^{n}\left(x_{1}\right)}{\tau}>0$, a contradiction. Similarly, if there exists $x_{1}>x_{0}$ such that $v^{n+1}\left(x_{1}\right)=v_{x}^{n+1}\left(x_{1}\right)=0, v_{x x}^{n+1}\left(x_{1}\right)>$ 0 and there is a unique simple zero on $\left[x_{0},+\infty\left[;\right.\right.$ if $v^{n+1}\left(x_{0}\right)=0$, there is no zero on $] x_{0},+\infty\left[; x_{0}\right.$ cannot be a double zero of $v^{n+1}$ : if not, $v_{x x}^{n+1}\left(x_{0}\right)=0$, and, since $v_{x}^{n}\left(x_{0}\right)<0, v_{x x x}^{n+1}\left(x_{0}\right)>0$ and $v^{n+1}$ is strictly increasing in a neighbourhood of $x_{0}$. Thus, it remains to study the number of zeros of $v^{n+1}$ in $\left[0, x_{0}[\right.$.

The maximum principle implies that

$$
v^{n+1} \geq \min \left(v^{n+1}(0), v^{n+1}\left(x_{0}\right), 0\right),
$$

and we have to distinguish once again the cases $v^{n+1}\left(x_{0}\right) \geq 0$ and $v^{n+1}\left(x_{0}\right)<0$. If the former occurs, $v^{n+1}(0) \neq 0$ : if $v^{n+1}(0)=0, v^{n+1}$ is decreasing in a vicinity of 0 , in contradiction with (3.6). Moreover, $v^{n+1}(0)$ cannot be negative. Indeed,

$$
\begin{aligned}
v_{x}^{n+1}(0) & =-\frac{\log R^{n+1}-\lambda\left(R^{n+1}\right)^{2}}{R^{n+1}-R_{1}} v^{n+1}(0)-\frac{E q^{n+1}}{R^{n+1}} \\
& :=\varphi^{n+1} v^{n+1}(0)-\frac{E q^{n+1}}{R^{n+1}}
\end{aligned}
$$

where $\varphi^{n}$ is bounded by $2|\log \tau| / R_{1}$. Let us define $w^{n}=e^{-x \varphi^{n}} v^{n}$. Then,

$$
\left\{\begin{array}{l}
w_{x x}^{n+1}+2 \varphi^{n+1} v_{x}^{n+1}+\left(\left(\varphi^{n+1}\right)^{2}-\frac{1}{\tau}\right) w^{n+1}=-\frac{e^{x\left(\varphi^{n}-\varphi^{n+1}\right)}}{\tau} w^{n} \leq 0, \\
w_{x}^{n+1}(0)=-\frac{E q^{n+1}}{R^{n+1}} \leq 0
\end{array}\right.
$$

The parameter $\lambda$ being fixed, fix $\tau>0$ small enough such that $\left(\varphi^{n+1}\right)^{2}-1 / \tau<0$. Then, $\left.w^{n+1} \geq \min \left(w^{n+1}(0), 0\right)\right)$. Yet, $w^{n+1}(0)>0$ by the Hopf lemma, and consequently, $v^{n+1}(0)>0$. Moreover, if $v^{n+1}$ vanishes on $\left[0, x_{0}[\right.$, we have a local non positive minimum in $\left.x_{1} \in\right] 0, x_{0}\left[\right.$, and $v_{x x}^{n+1}\left(x_{1}\right) \leq-v^{n}\left(x_{1}\right) / \tau<0$, a contradiction.

Using the same arguments, one checks easily that if $v^{n+1}\left(x_{0}\right)<0$, there is at most one zero on $\left[0, x_{0}[\right.$, which ends the study of the possibility of a simple zero of $v^{n}$ on $\left[0, x_{0}\left[\right.\right.$ if $x_{0}>0$.

Case 3: $v^{n}$ admits a simple or a double zero in 0 .

Then, $L v^{n+1} \geq 0$, and $\max v^{n+1} \leq \max \left(v^{n+1}(0), 0\right)$. As before, we cannot have any local non negative maximum or a double zero in $x_{1}>0$, and the unique point which has to be verified is the non-existence of a triple zero in 0. Indeed, $v^{n+1}(0)=v_{x}^{n+1}(0)=0$ implies $v_{x x}^{n+1}(0)=0$. Moreover, $\left(v^{n+1}\right)^{(3)}(0)=-\frac{v_{x}^{n}(0)}{\tau}$. If $v_{x}^{n}(0)<0$, 
$\left(v^{n+1}\right)^{(3)}(0)>0$; if $v_{x}^{n}(0)=0, v_{x x}^{n}(0)<0$ and $\left(v^{n+1}\right)^{(4)}(0)=-\frac{v_{x x}^{n+1}(0)}{\tau}>0$. In both cases, $v^{n+1}$ is increasing in a neighbourhood of 0 , which is impossible.

Let us finally notice that, by connectedness of the support of $q$, if 0 is a double zero for $v^{n}$, then $v^{n+1}<0$; if 0 is a simple zero for $v^{n}, 0$ is at most a simple zero for $v^{n+1}$.

Theorem 3.8 implies the following result:

Corollary 3.9. (i) $u^{n} \leq R_{1} \Leftrightarrow u^{n}(0) \leq R_{1}$.

(ii) Let the support of $q$ be the interval $] 0, t_{0}\left[, t_{0}<+\infty\right.$. Then, $E \in X_{-}$if and only if there exists $n \in \mathbb{N}^{*}$ such that $n \tau>t_{0}$ and $R^{n}<R_{1}$.

Proof.

(i) It is a direct consequence of Theorem 3.8.

(ii) Because of the results of convergence written in Theorem 2.2, there exists $\alpha<1$ and $n_{0} \in \mathbb{N}^{*}$ such that $u^{n_{0}} \leq \alpha<1$ and $n_{0} \tau>t_{0}$. Therefore, $u^{n_{0}+n} \leq v^{n}$ by Theorem 1.1, where:

$$
\left\{\begin{array}{l}
v_{x x}^{n+1}-\frac{v^{n+1}}{\tau}=-\frac{v^{n}}{\tau} \\
v_{x}^{n+1}(0)=-\log v^{n+1}(0)+\lambda\left(v^{n+1}(0)\right)^{2} \\
v^{0}=\alpha
\end{array}\right.
$$

and we conclude by Theorem 3.3 .

Proof of Proposition 3.7. First, let $E$ belong to $X_{+}$. There exists $n_{0}$ such that $\forall n \geq n_{0}, R^{n} \geq R_{\mathrm{cr}}$. According to Theorem 3.3, $u^{n}$ converges uniformly towards $R_{2}$ on every compact subset of $\mathbb{R}$. Thus, there exists $n_{1} \geq n_{0}$ such that $\forall n \geq n_{1}$,

$$
u_{E}^{n} \geq \frac{R_{2}+R_{\mathrm{cr}}}{2} \text { on }\left[\frac{-2 \sqrt{e}}{1-2 \lambda e}, \frac{2 \sqrt{e}}{1-2 \lambda e}\right] .
$$

Because of the continuity of $\left(u^{n}\right)$ with respect to $E$, for $E^{\prime}$ near enough to $E$,

$$
u_{E^{\prime}}^{n_{1}} \geq R_{\mathrm{cr}} \text { on }\left[\frac{-2 \sqrt{e}}{1-2 \lambda e}, \frac{2 \sqrt{e}}{1-2 \lambda e}\right] .
$$

Hence, $u_{E^{\prime}}^{n_{1}} \geq \underline{\phi}$, where $\underline{\phi}$, subsolution of (3.2), is the non-negative part of $\phi_{R_{\mathrm{cr}}}$, function defined in (3.4); therefore $\lim _{n \rightarrow+\infty} u_{E^{\prime}}^{n}=R_{2}$.

Let us now prove that $X_{-}$is open: when $q$ is compactly supported, it follows directly from Corollary 3.9 and the continuity of $R_{E}^{n}$ with respect to $E$. If $q(t)>0$ for all $t>0$, there exists $\varepsilon>0$ such that

$$
2 \varepsilon E-\log 2-\lambda / 4<0 .
$$

Let $n_{\varepsilon} \in \mathbb{N}^{*}$ such that $q^{n} \leq \varepsilon$ and $u^{n_{\varepsilon}} \leq 1 / 4$. Then, we infer the result from Theorems 1.1 and 3.3 and the continuity of $u_{E}^{n}$ with respect to $E$.

From now on, let us choose $E \in X_{0}$. Let now introduce, for every Lipschitz square-integrable function $u_{0}$, the $\omega$-limit set $\omega\left(u_{0}\right)$ of $u_{0}$ with respect to (1.9):

$$
\omega\left(u_{0}\right)=\left\{\psi \in \mathcal{C}^{\infty}\left(\mathbb{R}^{*}\right), \psi \neq 0, \exists \varphi(n) \in \mathbb{N}^{*}, \varphi(n) \nearrow+\infty, u^{\varphi(n)} \rightarrow \psi \text { when } n \rightarrow+\infty\right\} .
$$


Proposition 3.10. Let assume $\omega(0)$ to be non-empty. Then, there exists $\psi$ belonging to $\omega(0)$ satisfying one of the three possibilities:

(i) $\psi \equiv R_{1}$.

(ii) $\psi(x)>R_{1}, \forall x \in \mathbb{R}$.

(iii) $\psi(x)<R_{1}, \forall x \in \mathbb{R}$.

Proof. Because of Theorem 3.8, either $\psi-R_{1}$ has exactly two zeroes and $\psi(0)>R_{1}$, or $\psi \leq R_{1}$ and the only zero of $\psi-R_{1}$ is 0 . If the latter occurs, let us consider $\left(u^{n}\right)$ defined by:

$$
\left\{\begin{array}{l}
u_{x x}^{n+1}-\frac{u^{n+1}}{\tau}=-\frac{u^{n}}{\tau}, x>0 \\
u_{x}^{n+1}(0)=-\log u^{n+1}(0)+\lambda u^{n+1}(0)^{2} \\
u^{0}=\psi
\end{array}\right.
$$

This problem has a solution for $n>0$, and on the life set of $u^{n}, u^{n}<R_{1}$. Indeed, let $v^{n}=u^{n}-R_{1}$, and assume $v^{n}<0$. Let us now prove that $v^{n+1}<0$.

$$
\left\{\begin{array}{l}
v_{x x}^{n+1}-\frac{v^{n+1}}{\tau}=-\frac{v^{n}}{\tau} \geq 0 \\
v_{x}^{n+1}(0)=-\log u^{n+1}(0)+\lambda u^{n+1}(0)^{2}
\end{array}\right.
$$

By the maximum principle, $\max _{[0,+\infty[} v^{n+1} \leq \max \left(v^{n+1}(0), 0\right)$. We cannot have $v^{n+1}(0)>0$. Indeed, if $f^{n}$ denotes the function defined in the first section,

$$
u^{n+1}(0)-u^{n}(0)=\sqrt{\tau} f^{n+1}\left(u^{n+1}(0)\right)-\tau \int_{\mathbb{R}} \frac{4 \pi \xi^{2}}{1+4 \pi^{2} \xi^{2} \tau} \hat{u}^{n}(\xi) \mathrm{d} \xi
$$

so that $u^{n+1}(0)-u^{n}(0)$ converges uniformly to 0 as $\tau$ tends to 0 , as long as $u^{n}(0) \geq R_{1}$. Thus, for $\tau$ small enough and $\lambda$ not too close of $\lambda_{\mathrm{cr}}, R_{1} \leq u^{n+1}(0) \leq R_{2}$ and $v_{x}^{n+1}(0) \geq 0$, in contradiction with the Hopf lemma. If $v^{n+1}(0)=0, v_{x}^{n+1}(0)=0$ and $v_{x x}^{n+1}(0)>0$; this contradicts Theorem 3.8. Thus, Theorem 3.8 applies once again and $u^{n}<R_{1}$, which gives the existence of $\tilde{\psi} \in \omega(0)$ satisfying $(i)$ or $(i i i)$.

Let us turn now to the former case: according to Corollary 3.9, $\psi^{n}$ is bounded, and bounded away from 0 . Therefore, $\omega(\psi)$ is non empty and $\omega(\psi) \subset \omega(0)$ since $\psi \in \omega(0)$. Let $x_{i}^{n}$ be the two zeroes of $\psi^{n}$. Either $0 \in \overline{\left\{x_{i}^{n}\right\}}$ and there exists $\tilde{\psi}$ belonging to $\omega(\psi)$ and checking $(i)$ or $($ iii $)$. Or, $\left(x_{i}^{n}\right)$ is bounded away from 0 and we can find a subsolution $\phi$ of (3.2) such that $\phi \leq \psi$. Then, we conclude by Theorem 3.3 and we infer the existence of $\tilde{\psi} \in \omega(\psi)$ satisfying $(i)$ or $(i i)$.

Remark. let us just notice here the dependence on $\tau$ of $\lambda_{\mathrm{cr}}(\tau)$, which appears in this proof. Moreover, we have $\lim _{\tau \rightarrow 0} \lambda_{\mathrm{cr}}(\tau)=\lambda_{\mathrm{cr}}$ because of the convergence result written in Proposition 2.2.

If $R_{1} \in \omega(0)$, then the sequence $\left(R^{n}\right)_{n}$ obviously converges towards $R_{1}$. Therefore, it remains to check that cases $($ ii $)$ or $($ iii $)$ may not happen. To do so, for any $L$ positive, let us consider the semi-group $\left(S_{L}^{n}\right)$ defined by:

$$
\left\{\begin{array}{l}
\left.u_{x x}^{n+1}-\frac{u^{n+1}}{\tau}=-\frac{u^{n}}{\tau}-2 \delta_{x=0}\left(\log u^{n+1}-\lambda\left(u^{n+1}\right)^{2}\right), x \in\right]-L, L[ \\
u^{n+1}( \pm L)=R_{1} \\
u^{0}=u_{0}
\end{array}\right.
$$

Proposition 3.11. There exists $L_{0}>0$ such that for all $L>L_{0}, S_{L}^{n}$ has two fixed points: the unstable solution $R_{1}$ and a stable solution $\psi_{L}^{+}>R_{1}$. Moreover, $\lim _{L \rightarrow+\infty} \psi_{L}^{+}=R_{2}$. Let $u_{0} \in \mathcal{C}^{1}([-L, L])$ an even function which checks $u_{0}( \pm L)=R_{1}, u(0) \neq R_{1}$. If $u_{0} \geq R_{1}, S_{L}^{n} u_{0} \rightarrow \psi_{L}^{+}$on every compact subset of $]-L, L[$. 
The proof of this proposition is omitted, being the exact equivalent to the one written in [12]. This proposition will apply when $q$ is compactly supported. When $q>0$ on $\mathbb{R}_{+}^{*}$, we have a similar result, whose proof is also omitted: let $\left(S_{L, \varepsilon}^{n}\right)$ be the semi-group associated to:

$$
\left\{\begin{array}{l}
\left.u_{x x}^{n+1}-\frac{u^{n+1}}{\tau}=-\frac{u^{n}}{\tau}-2 \delta_{x=0}\left(\log u^{n+1}+\frac{\varepsilon}{u^{n+1}}-\lambda\left(u^{n+1}\right)^{2}\right), x \in\right]-L, L[ \\
u^{n+1}( \pm L)=R_{1} \\
u^{0}=u_{0}
\end{array}\right.
$$

Proposition 3.12. For $L$ large enough, $\left(S_{L, \varepsilon}^{n}\right)$ has three fixed points: $\psi_{L, \varepsilon}^{-}<\psi_{L, \varepsilon}^{0}<\psi_{L, \varepsilon}^{+}$. The fixed points $\psi_{L, \varepsilon}^{-}$ and $\psi_{L, \varepsilon}^{+}$are stable, although $\psi_{L, \varepsilon}^{0}$ is unstable. Moreover,

$$
\lim _{\varepsilon \rightarrow 0} \psi_{L, \varepsilon}^{-}=\psi_{L}^{-}, \lim _{\varepsilon \rightarrow 0} \psi_{L, \varepsilon}^{0}=R_{1}, \lim _{\varepsilon \rightarrow 0} \psi_{L, \varepsilon}^{+}=\psi_{L}^{+}
$$

these limits being uniform on $[-L, L] . \psi_{L, \varepsilon}^{+}\left(\right.$resp. $\left.\psi_{L, \varepsilon}^{-}\right)$attracts all the trajectories starting above (resp. below) $\psi_{L, \varepsilon}^{0}$.

We can now turn to the characterization of $X_{0}$.

Theorem 3.13. The set $X_{0}$ is reduced to one point $E_{\mathrm{cr}}$ and $\lim _{n \rightarrow+\infty} R_{E_{\mathrm{cr}}}^{n}=R_{1}$.

Proof. Let us first prove that for $E \in X_{0}, R_{E}^{n} \rightarrow R_{1}$. We have to distinguish $q$ compactly supported from $q^{n}$ positive $\forall n \in \mathbb{N}^{*}$.

Case 1: The function $q$ is compactly supported.

We know that $u_{E}^{n}$ is bounded, and $R_{E}^{n}$ is bounded away from 0 because of Corollary 3.9. So, $\omega(0) \neq \emptyset$. Let $\psi$ belong to $\omega(0)$, and assume that $\psi$ satisfies the assertion $(i i)$ of Proposition 3.10. Let $L>1$ be large enough so that Proposition 3.11 is valid. The function $\underline{u}^{n}$, defined by:

$$
\left\{\begin{array}{l}
\underline{u}^{n}=S_{L}^{n} \psi \text { if } x<L \\
\underline{u}^{n}=R_{1} \text { if } x \geq L
\end{array}\right.
$$

satisfies: $\lim _{L \rightarrow+\infty, n \rightarrow+\infty} \underline{u}^{n}=R_{2}$ and $\underline{u}^{n} \leq S^{n} \psi$. So, for $n$ large enough, there exists $\left.\phi_{b}, b \in\right] R_{1}, R_{2}[$ such that $u_{E}^{n}>\phi_{b}$, hence $\lim _{n \rightarrow+\infty} u_{E}^{n}=R_{2}$. If $\psi$ satisfies $(i i i)$, there exists $n_{0} \in \mathbb{N}^{*}$ such that $q\left(n_{0} \tau\right)=0$ and $R_{E}^{n_{0}}<1$. By Corollary 3.9, we know that $\lim _{n \rightarrow+\infty} u_{E}^{n}=0$.

Case 2: $q>0$ on $\mathbb{R}_{+}^{*}$.

Let us prove that $R_{E}^{n}$ is bounded away from 0 . Because of the previous analysis, we will have $\omega(0)=\left\{R_{1}\right\}$, and the proof will be finished. Let us assume the existence of $\varphi(n) \in \mathbb{N}, \varphi(n) \nearrow+\infty$, such that $R_{E}^{\varphi(n)}<R_{1}$. Theorem 3.8 asserts that $u_{E}^{n}<1$ and $\forall n$, there exists $\alpha<R_{1}$ such that $u_{E}^{\varphi(n)+1} \leq \alpha$. For $n$ sufficiently large, let $\varepsilon$ positive small enough so that Proposition 3.12 applies with $L>1$ and $\psi_{L, \varepsilon}^{0}>\alpha$. Then, because

$$
R_{E}^{n} \leq S_{L, \varepsilon}^{m-\varphi(n)-1} u_{E}^{\varphi(n)+1} \quad \forall m \geq \varphi(n)+1,
$$

we get $S_{L, \varepsilon}^{m-\varphi(n)-1} u_{E}^{\varphi(n)+1}=O(\varepsilon)$ when $m \rightarrow+\infty$, and so $R_{E}^{n}=O(\varepsilon)$ when $n \rightarrow+\infty$. Hence, $E \in X_{-}$.

It remains to prove that $X_{0}$ is reduced to one point, but this is a stability argument similar to [1].

Remark. The threshold phenomenon obtained on the numerical scheme, Corollary 3.9 and the convergence result presented in the previous part imply that

$$
\lim _{\tau \rightarrow 0} E_{\mathrm{cr}}(\tau, q)=E_{\mathrm{cr}}(q) .
$$




\section{Presentation of Numerical Results AND COMPARISON With OtheR Methods}

In the forthcoming, we present numerical results, performed with an input energy $q=\chi_{[0,1]}$. In a first series of runs, we have observed the characteristic features of the flame evolution, according to the parameters $E$ and $\lambda$. On the one hand, we have chosen $\lambda=0.1<\lambda_{\mathrm{cr}}$, and we have recovered in Figure 1 the expected behaviour of the radius: when $E$ is small, the flame quenches; when it is larger, the behaviour cannot be guessed with this time scale, and numerical simulations have to be performed for long times for these energies, which has been done in Figure 2: the flame stabilizes towards the critical radius $R_{2}$. When $\lambda$ is variable for $E$ fixed, results are similar (cf. Fig. 3): quenching for the more important heat losses, and stabilization to the corresponding critical radius, depending on the value of $\lambda$.

On the other hand, we have compared this method with others: indeed, in [8], numerical simulations have been performed from an implicit method based on an approximation of $\dot{R}$ by piecewise constant or linear

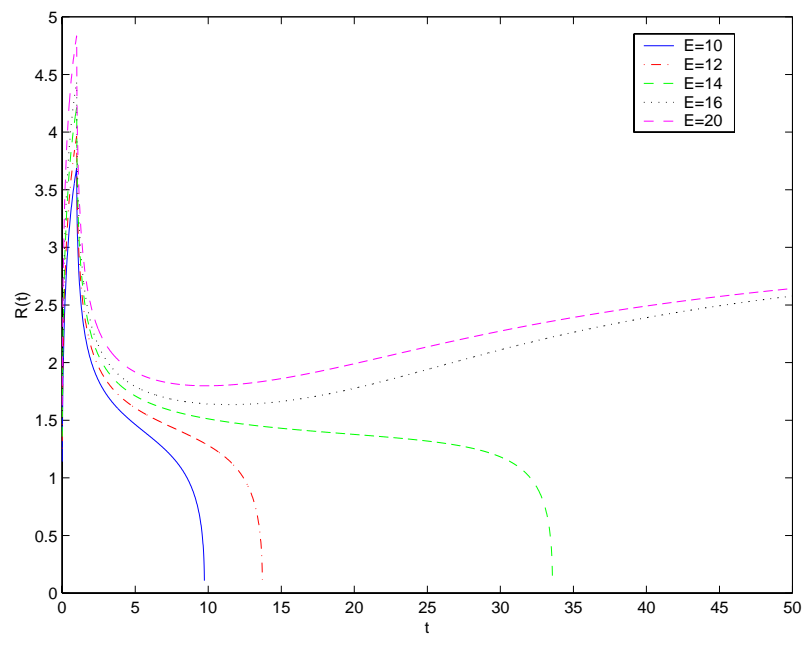

FiguRE 1. Evolution of the radius when $\lambda<\lambda_{\text {cr }}$ and $E$ variable.

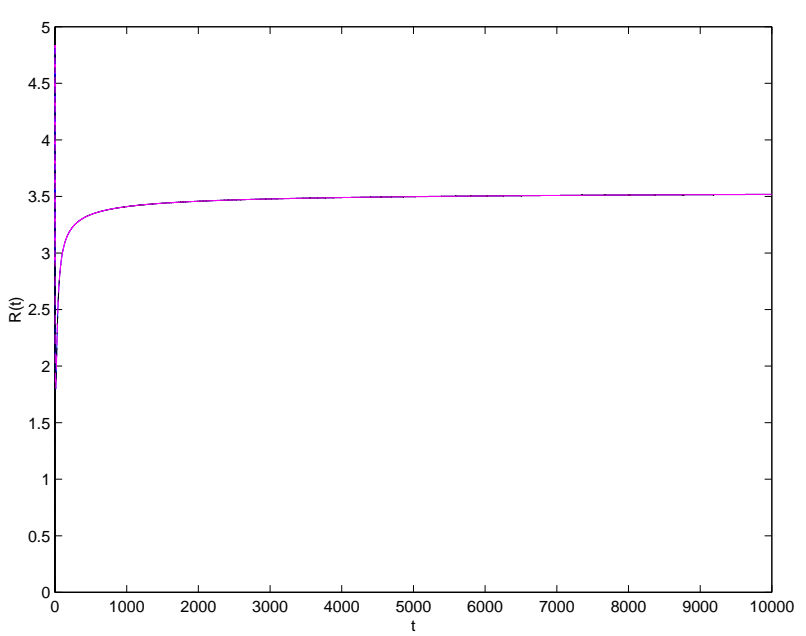

Figure 2. Stabilization towards $R_{2}$ when $E>E_{\mathrm{cr}}$.

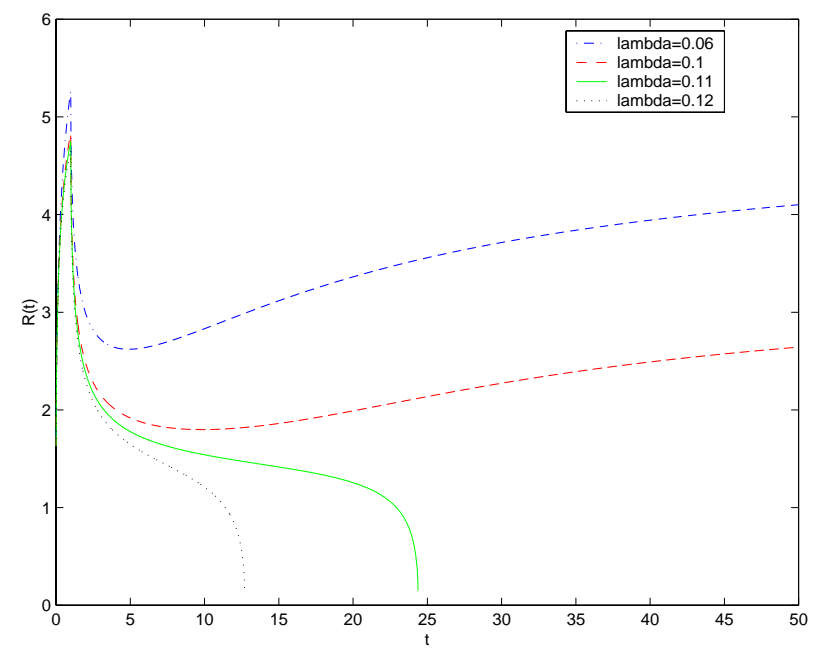

Figure 3. Evolution of the radius when $E$ fixed and $\lambda$ variable. 

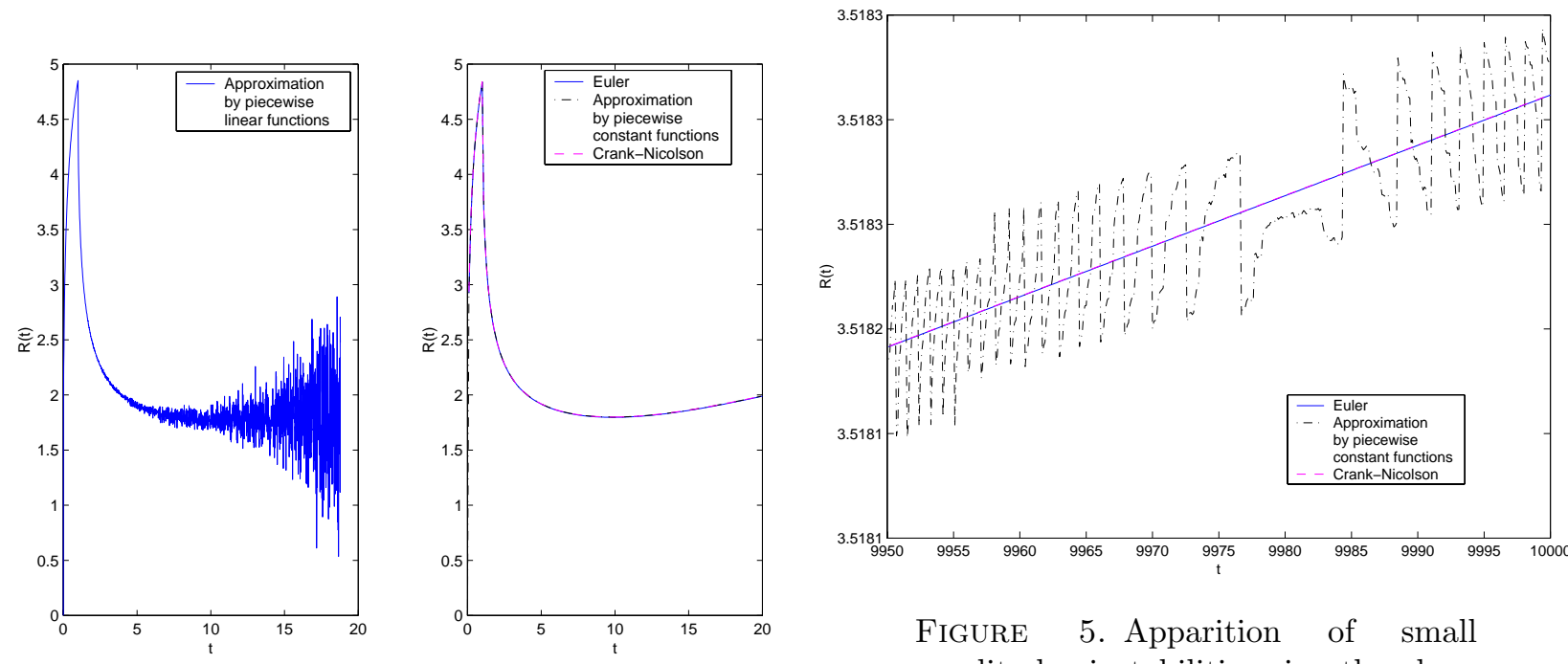

Figure 5. Apparition of small amplitude instabilities in the long times for the approximation by piecewise constant functions.

Figure 4. Initiation of the flame: comparison between all the methods.

functions. We want to compare here these methods with the method described above. In order to increase its order, we have also done a time-discretisation of (1.2), based on a Crank-Nicolson scheme. We can solve it in the same way as done in the first part, which leads to the following recursive formula:

$$
u^{n}(0)=\sqrt{2 \tau} \sum_{k=1}^{n-1} \gamma_{k} g^{n-k+1},
$$

where $g^{n}=f^{n}\left(\frac{u^{n-1}(0)+u^{n}(0)}{2}\right), \gamma_{2 k}=0, \gamma_{2 k+1}=(1-1 / 2 k) \gamma_{2 k-1}$ and $\gamma_{1}=1$. The function $f^{n}$ is the one defined in the first section. The values of $\gamma_{k}$ are obtained thanks to the Wallis integrals. This method is not studied here. Indeed, to conserve the positivity of the heat equation by discretisation, we have to use schemes of order one (see [3]).

At first, we compare their performances with $\lambda=0.1<\lambda_{\mathrm{cr}}$ and $E=20>E_{\mathrm{cr}}(q)$. The approximation in a space of piecewise linear functions, presented in Figure 4, fails; but, the three other methods give comparable results, as much with the quantitative results than with the time calculation. Nevertheless, it has to be noticed that small spurious instabilities appear in long times by using the method described in [8] (cf. Fig. 5). In particular, it does not conserve the positive properties of the integro-differential equation, unlike the results observed with Crank-Nicolson and Euler, which use is more interesting when one wants to observe the stabilization towards the critical radius.

For energies close to the critical energy, the results obtained by these three methods are different, as seen in Figure 6: the flame quenches by the use of the approximation by piecewise constant functions and the Euler implicit scheme - but with a different quenching time - whereas the flame stabilizes with the CrankNicolson scheme: in a vicinity of the critical energy, these numerical methods are extremely sensitive to slight perturbations. This phenomenon is illustrated in Figure 7: the numerical solution, obtained with Euler scheme, has been replaced by the ansatz $R_{0} t^{1 / 4}$ (equivalent of the radius at $t=0[1]$ ) during very short times. While the numerical solution quenches with the classical Euler scheme, the solution computed with the ansatz stabilizes with this small change in the first times.

We hope, in a future study, to devise numerical schemes for flame ball models accounting for more realistic situations, such as heat loss in the whole domain, heat loss by turbulent convection... 


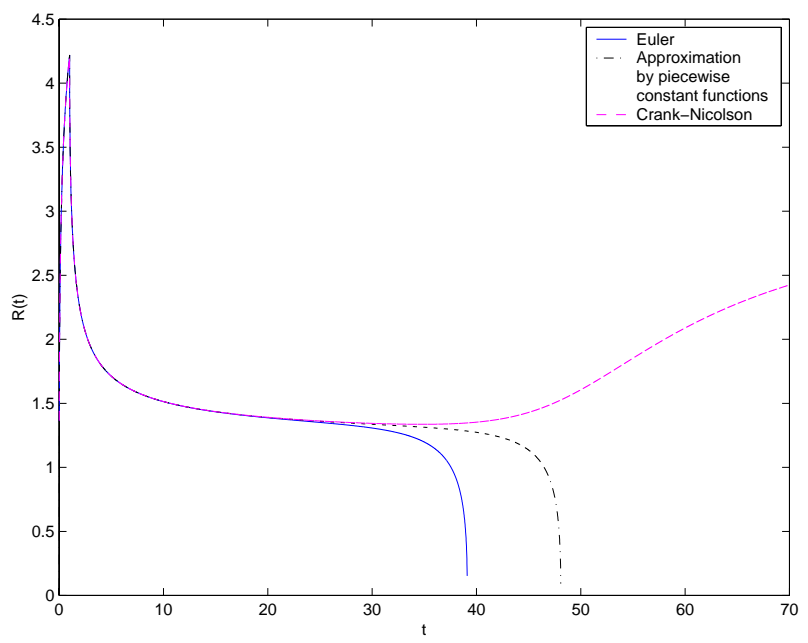

FIGURE 6. Evolution of the radius for any energy close to the critical energy for the three methods

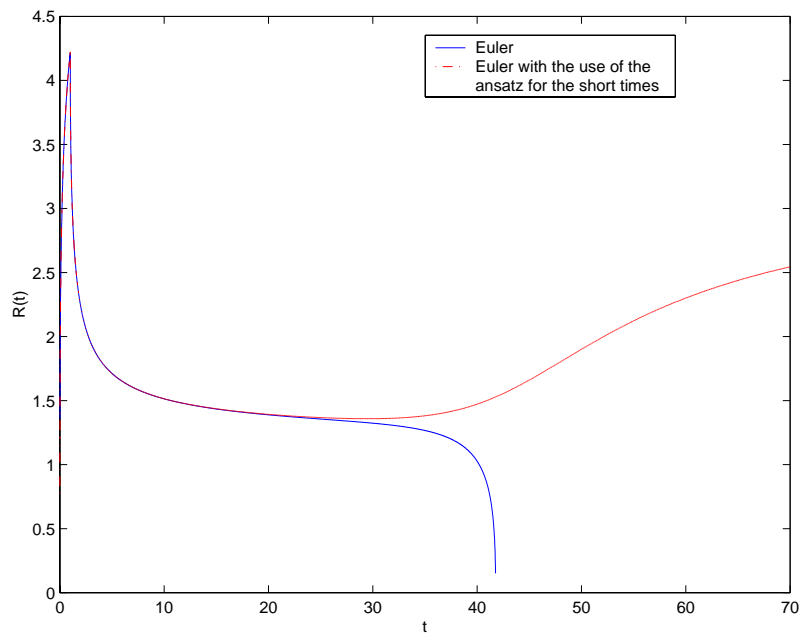

FiguRE 7. Use of the ansatz of the radius in the very first times

\section{REFERENCES}

[1] J. Audounet, V. Giovangigli and J.-M. Roquejoffre, A threshold phenomenon in the propagation of a point source initiated flame. Phys. D 121 (1998) 295-316.

[2] J. Audounet and J.-M. Roquejoffre, An integral equation describing the propagation of a point source initiated flame: Asymptotics and numerical analysis. Systèmes différentiels fractionnaires: Modèles, Méthodes et Applications, Matignon \& Montseny Eds, ESAIM Proc. 5 (1998).

[3] C. Bolley and M. Crouzeix, Conservation de la positivité lors de la discrétisation des problèmes d'évolution paraboliques. RAIRO Anal. Numér. 3 (1978) 237-245.

[4] H. Brunner, A survey of recent advances in the numerical treatment of Volterra integral and integro-differential equations. $J$. Comput. Appl. Math. 3 (1982) 213-229.

[5] J. Buckmaster, G. Joulin and P. Ronney, The structure and stability of nonadiabatic flame balls. Combust. Flame 79 (1990) 381-392.

[6] J. Buckmaster, G. Joulin and P. Ronney, The structure and stability of nonadiabatic flame balls. II. Effects on far-field losses. Combust. Flame 84 (1991) 411-422.

[7] R. Gorenflo and S. Vessella, Abel Integral Equations. Analysis and Applications. Springer-Verlag, Berlin (1991).

[8] G. Joulin, Point source initiation of lean spherical flames of light reactants: An asymptotic theory. Combust. Sci. Tech. 43 (1985) 99-113.

[9] O.A. Ladyzhenskaya, N.N. Uraltseva and S.N. Solonnikov, Linear and quasilinear equations of parabolic type. Transl. Math. Monogr. 23 (1968).

[10] C. Lubich, Discretized fractional calculus. SIAM J. Math. Anal. 3 (1986) 704-719.

[11] C. Lubich and A. Ostermann, Linearly implicit time discretization of non-linear parabolic equations. IMA J. Numer. Anal. 15 (1995) 555-583.

[12] H. Rouzaud, Dynamique d'un modèle intégro-différentiel de flammes sphériques avec pertes de chaleur. C.R. Acad. Sci. Paris Sér. 1332 (2001) 1083-1086. 